\title{
Perfil dos indivíduos que iniciam programas de exercícios em academias, quanto à composição corporal e aos objetivos em relação a faixa etária e sexo
}

\author{
Ronaldo Domingues Filardo ${ }^{1}$ e Neiva Leite ${ }^{2}$
}

\section{RESUMO}

A busca de melhor qualidade de vida nos últimos anos faz aumentar o número de pessoas preocupadas com a redução do peso corporal. Muitos indivíduos procuram atividades físicas regulares como forma alternativa de tratamento. O objetivo deste estudo foi investigar o perfil dos indivíduos que iniciam programas de exercícios em academias, quanto à composição corporal e aos objetivos em relação à faixa etária e ao sexo. Pesquisa transversal, com amostragem de 90 homens e 89 mulheres, composta por alunos iniciantes de uma academia em Curitiba, avaliados entre agosto de 1998 e setembro de 1999. Para a coleta de dados os alunos preencheram uma ficha que continha objetivos predeterminados. As medidas antropométricas, massa corporal, estatura e dobras cutâneas seguiram as indicações de Wartenweiler et al. (1974). No cálculo da densidade corporal em adultos utilizou-se a fórmula de Petroski (1995) - 4 dobras cutâneas. Para a estimativa do percentual de gordura $(\% \mathrm{G})$ em adultos a fórmula de Siri (1961). O tratamento estatístico foi com o teste "t" de Student para grupo independentes. Foram observadas diferenças significativas ( $\mathrm{p}<0,05)$, para o índice de massa corporal (IMC) e \% G nos homens, e, para \% G nas mulheres entre as faixas etárias. Para os indivíduos de 20 a 30 anos, 45,7\% dos homens desejam aumentar massa muscular; $54,9 \%$ das mulheres esperam reduzir peso. Entre 30 e 40 anos, 35\% dos homens desejam redução de peso corporal. Embora $55 \%$ das mulheres entre 20 e 30 anos desejem redução de

1. Especialista em Exercício e Qualidade de Vida pela UFPR.

2. Mestre em Reabilitação pela UFSP.

Recebido em: 16/8/2000.

Aceito em: 7/3/2001.

Endereço para correspondência:

Ronaldo Domingues Filardo

Rua Francisco Xavier Oliveira, 525

81950-070 - Curitiba, PR

Fone: (41) 9102-6435

E-mail: ronaldofilardo@zipmail.com.br peso corporal, somente $16,9 \%$ delas realmente necessitam. Pode-se concluir que o incremento da gordura corporal ocorre em ambos os sexos com o avanço da idade e que a análise dos valores médios de \% $\mathrm{G}$ e IMC indicam que homens e mulheres têm sua condição de saúde agravada e seus objetivos modificados com a idade.

Palavras-chave: Composição corporal. Atividade física. Programas de exercício.

\section{ABSTRACT}

Profile of individuals who starts exercise programs in gyms, as to body composition and goals in relation to age and sex

The search for a better quality of living in the recent years has increased the number of people concerned about body weight reduction. Many are looking for regular physical activities as an alternative way of treatment. The goal of this study was to investigate the profile of people entering a physical exercise program in fitness clubs, regarding body composition and purposes according to age and sex. A cross-sectional study, with a sample of 90 men and 89 women, who were physical activity beginners in a fitness club in Curitiba, evaluated between August 1998 and September 1999 was performed. To collect data, subjects were asked to fill in a questionnaire with preset goals. Data concerning anthropometric measures, weight, height, and skinfolds were collected according to Wartenweiler et al. (1974). To estimate the adult body density, the four skinfolds equation of Petroski (1995) was used. To estimate the percentage of body fat (\%F) the Siri (1961) equation was used. The Student $t$-test for independent samples was the statistical treatment used. There was a significant statistical difference ( $p<0.05)$ between age groups for body mass index (BMI) and \%F in men and for \%F in women. It was also observed that $45.7 \%$ of men with age between 20 and 30 years wished to increase their muscle mass and $54.9 \%$ of the women wanted to reduce their weight. For the age groups between 30 and 40 years, 35\% of the men wished 
to reduce weight. Although 55\% of the women between 20 and 30 years wished to reduce body weight, only 16.9\% really needed it. The authors concluded that body fat increased with age in both male and female and that the mean values of \%F and BMI showed that men and women have health conditions impaired with aging along with a change in their goals.

Key words: Body composition. Physical activity. Exercise programs.

\section{INTRODUÇÃO}

Um dos objetivos mais freqüentes na procura do exercício físico é a redução de peso corporal. Sabe-se que o excesso de peso está associado a inúmeras condições clínicas (também consideradas fatores de risco para doenças crônicas degenerativas), tais como obesidade, hipertensão, diabetes, taxas plasmáticas de colesterol e triglicerídeos anormais entre outros. Anualmente milhões de indivíduos são submetidos a programas de redução de peso por numerosas razões; conseqüentemente, a redução de peso é com frequiência recomendada pelos médicos por razões clínicas; além disso, motivos estéticos também levam grande número de indivíduos a esses programas ${ }^{1}$.

A associação de fatores de risco com vários estados patológicos já está comprovada ${ }^{2}$, sendo que o fator de risco que mais cresce é a obesidade. Esta é o distúrbio nutricional mais importante do mundo desenvolvido; entre outros fatores o sedentarismo desempenha papel fundamental na indução e manutenção do peso corporal, sendo que indivíduos sedentários apresentam percentual de gordura corporal maior do que os que se exercitam freqüentemente ${ }^{3}$.

Em estudo relacionando o nível de adesão ao exercício para programa supervisionado, Nunomura et al. ${ }^{4}$ observaram que $25 \%$ dos praticantes desistem das aulas por acharem os horários destas inadequados às suas necessidades (principal motivo), 14\% por acharem o nível das aulas muito fraco; os autores também relataram um nível de resistência à mudança de comportamento muito alto nestes participantes, fazendo com que o insucesso destes programas aconteça naturalmente.

No Brasil, estudos sobre composição corporal que envolvam sujeitos adultos com amostragem suficiente para a generalização de resultados são raros, talvez pela extensão territorial ${ }^{5}$ ou falta de centros de pesquisas específicos da área. Contudo, estudos regionais ${ }^{6-8}$ vêm demonstrando a importância dessas pesquisas para o perfil do brasileiro, em relação aos inúmeros fatores de risco à saúde. Na cidade de Curitiba pouco tem sido feito e publicado, o que vem demonstrar a necessidade de suprir esta enorme lacuna relacionada ao comportamento e perfil de saúde dessa população.
Desta maneira, investigar o perfil dos indivíduos que iniciam programas de exercícios em academias, quanto à composição corporal e objetivos em relação à faixa etária e sexo foi o principal intento deste trabalho.

\section{PROCEDIMENTOS METODOLÓGICOS}

Estudo transversal descritivo, sendo a amostra composta de 179 sujeitos (90 homens e 89 mulheres), alunos iniciantes de programas de exercícios em uma academia da cidade de Curitiba, no período de agosto de 1998 a setembro de 1999. Os avaliados foram divididos em faixas etárias, 1) de 20 a 30 anos; 2) de 30,1 a 40 anos. Os dados demográficos são apresentados nas tabelas 1 e 2 .

$\mathrm{O}$ instrumento de coleta de dados foi uma ficha, composta por seis objetivos que os alunos escolhiam conforme a sua preferência quanto à prescrição, sendo: $\mathrm{n}^{0}$ 1) reduzir peso e/ou emagrecimento: composto por uma maior carga de exercícios aeróbios; $\mathrm{n}^{\circ}$ 2) aumento de massa muscular: para pessoas com percentual de gordura próximo ao ideal; $\mathrm{n}^{\circ} 3$ ) tonificação geral: para praticantes que necessitassem

\begin{tabular}{lcrlrrr}
\hline \multicolumn{7}{c}{ TABELA 1 } \\
Médias e desvios padrões da composição \\
corporal em homens, conforme a faixa etária \\
\hline Idade & MC & Estatura & IMC & \%G \\
20-30 anos & $\bar{X}$ & 24,1 & 75,5 & 176,8 & $24,1^{*}$ & $14,4^{* *}$ \\
$\mathrm{n}=70$ & $\mathrm{~S}$ & 2,8 & 11,0 & 6,9 & 2,9 & 3,7 \\
30-40 anos & $\bar{X}$ & 34,4 & 80,5 & 175,1 & $26,1^{*}$ & $18,7^{* *}$ \\
$\mathrm{n}=20$ & $\mathrm{~S}$ & 3,1 & 13,7 & 6,3 & 3,6 & 3,7 \\
Amostra total & $\bar{X}$ & 26,4 & 76,6 & 176,4 & 24,6 & 15,4 \\
& $\mathrm{~S}$ & 5,1 & 11,7 & 6,8 & 3,1 & 4,1 \\
\hline
\end{tabular}

* diferença significativa no IMC para $\mathrm{p}<0,05$

** diferença significativa no \%G para $p<0,05$

TABELA 2

Médias e desvios padrões da composição corporal em mulheres, conforme a faixa etária

\begin{tabular}{lcrrrrc}
\hline & & Idade & MC & Estatura & IMC & \%G \\
20-30 anos & $\bar{X}$ & 24,5 & 59,8 & 163,0 & 22,5 & $23,1^{*}$ \\
$\mathrm{n}=71$ & $\mathrm{~S}$ & 2,7 & 8,8 & 4,9 & 3,1 & 4,4 \\
30-40 anos & $\bar{X}$ & 34,2 & 63,0 & 162,7 & 23,7 & $26,8^{*}$ \\
$\mathrm{n}=18$ & $\mathrm{~S}$ & 2,9 & 11,3 & 7,2 & 3,4 & 5,3 \\
Amostra & $\bar{X}$ & 26,4 & 60,4 & 162,9 & 22,7 & 23,8 \\
& $\mathrm{~S}$ & 4,7 & 9,4 & 5,4 & 3,2 & 4,8 \\
\hline
\end{tabular}

* diferença significativa no $\% G$ para $p<0,05$ 
QUADRO 1

Equações de estimativa de densidade corporal em adultos

\begin{tabular}{|c|c|}
\hline Masc & $\mathrm{D}=1,10726863-(0,00081201 *(X 1))+\left(0,00000212^{*}(X 1)^{\wedge} 2\right)-(0,00041761 *$ idade $)$ \\
\hline Fem & $\begin{array}{l}D=1,02902361-(0,00067159 *(X 1))+(0,00000242 *(X 1) \wedge 2)-(0,00026073 * \text { idade })- \\
(0,00056009 * \text { peso })+(0,00054649 * \text { estatura })\end{array}$ \\
\hline$X 1=2$ & $\mathrm{DC} \mathrm{SE}+\mathrm{TRI}+\mathrm{SI}+\mathrm{PAM}$ \\
\hline
\end{tabular}

melhor condição de turgor muscular; n 4) saúde: para casos de indicação médica e patológicos; $\mathrm{n}^{\circ}$ 5) condicionamento físico generalizado: para recuperação de condição mínima objetivando prática de esportes no lazer, e no 6 ) outros: para praticantes com objetivos de esportes de rendimento, maior convívio social ou simplesmente lazer. Após esta etapa era solicitado o consentimento do avaliado para a utilização das informações em estudos futuros; em caso de negativa as fichas eram imediatamente excluídas da base de dados.

Foram medidos massa corporal (MC, kg), utilizando balança antropométrica (marca Filizola), estatura (EST, cm), com estadiômetro e dobras cutâneas (DC, mm) - com compasso (marca Cescorf) - tricipital (TRI), subescapular (SE), suprailíaca (SI) e panturrilha medial (PAM), seguindo as indicações de Wartenweiler et al. (1974) ${ }^{9}$.

Para o IMC foi utilizada a fórmula: Massa Corporal (kg)/ Estatura $^{2}(\mathrm{~m})$.

O protocolo para estimar a densidade corporal foi o proposto por Petroski ${ }^{10}$, conforme o quadro 1; e, para o cálculo do Percentual de Gordura $(\% \mathrm{G})$ foi utilizada a fórmula de Siri $(1961)^{11}$, em que \%Gord = (495/D)-450.

Para a análise dos dados utilizou-se a estatística descritiva e o teste t para grupos independentes, sendo considerado diferença estatisticamente significativa $\mathrm{p}<0,05$.

\section{RESULTADOS}

Nas tabelas 1 e 2, são apresentados os valores de indicadores antropométricos e de composição corporal, dos grupos e amostra total, em homens e mulheres, respectivamente. Para a amostra masculina detectou-se na faixa etária entre 20 e 30 anos somente maior estatura, em relação ao grupo de 30 a 40 anos. Foram observadas diferenças significativas para o Índice de Massa Corporal (IMC) e gordura relativa $(\% \mathrm{G})$.

$\mathrm{Na}$ amostragem feminina (tabela 2), observou-se padrão de comportamento dos indicadores antropométricos e da composição corporal semelhante aos homens, observan- do-se significância das diferenças somente para a gordura relativa.

Na tabela 3, é apresentada a distribuição percentual (frequiência relativa) dos indicadores de saúde (IMC e \%G). Pode-se observar um padrão relativamente inverso para os homens entre 20 e 30 anos, para o \%G e o IMC, ou seja, para o IMC existe maior percentual acima do ideal, e, para a gordura relativa, a maioria está abaixo do indicado. Ainda foi detectado que $10 \%$ dos homens entre 20 e 30 anos estão abaixo do IMC ideal; mas, $41,4 \%$ estão acima do indicado. Para a gordura relativa 38,5\% entre 20 e 30 anos têm menos do que $13 \%$ de gordura; acima de $17 \%$ de gordura, $21,5 \%$. Para a segunda faixa etária, houve percentuais iguais para a distribuição de IMC e \%G.

Ainda na tabela 3, na faixa etária de 30 a 40 anos 44,4\% do grupo feminino estão acima do esperado para o IMC e $\% \mathrm{G}$, ocorrendo inversão de percentuais para o IMC e \%G quando estes eram ideais e abaixo do indicado.

Na tabela 4, é apresentada a distribuição percentual de praticantes por sexo em relação à faixa etária para cada objetivo. Pode ser observado que mais homens na faixa etária de 20 a 30 anos se interessam em aumento de massa muscular $(45,7 \%)$ e, entre as mulheres, $54,9 \%$ em reduzir peso; entre 30 e 40 anos $35 \%$ dos homens desejam redu-

\begin{tabular}{|c|c|c|c|c|c|c|c|}
\hline \multicolumn{8}{|c|}{$\begin{array}{c}\text { TABELA } 3 \\
\text { Distribuição percentual dos praticantes por sexo } \\
\text { e faixa etária em relação aos valores de IMC e \%G }\end{array}$} \\
\hline \multirow[b]{2}{*}{ Sexo } & \multirow[b]{2}{*}{ FE } & \multicolumn{3}{|c|}{ IMCA } & \multicolumn{3}{|c|}{ \%GB } \\
\hline & & $<$ & Ideal & $>$ & $<$ & Ideal & $>$ \\
\hline Masculino & $\begin{array}{l}20-30 \\
30-40\end{array}$ & 10 & $\begin{array}{l}48,6 \\
40\end{array}$ & $\begin{array}{l}41,4 \\
60\end{array}$ & 38,5 & $\begin{array}{l}40 \\
40\end{array}$ & $\begin{array}{l}21,5 \\
60\end{array}$ \\
\hline Feminino & $\begin{array}{l}20-30 \\
30-40\end{array}$ & $\begin{array}{l}21,1 \\
16,7\end{array}$ & $\begin{array}{l}59,2 \\
38,9\end{array}$ & $\begin{array}{l}19,7 \\
44,4\end{array}$ & $\begin{array}{l}50,7 \\
38,9\end{array}$ & $\begin{array}{l}32,4 \\
16,7\end{array}$ & $\begin{array}{l}16,9 \\
44,4\end{array}$ \\
\hline
\end{tabular}

$A=$ Para IMC em ambos os sexos faixa ideal entre 20 e $24,9 \mathrm{~kg} / \mathrm{m} 2$, conforme Petroski ${ }^{8}$ $\mathrm{B}=$ Para o $\% \mathrm{G}$ ideal em homens entre 13 e $17 \%$, nas mulheres entre 23 e $27 \%$ 
ção de peso corporal, além de $30 \%$ desejarem outros objetivos ( $\mathrm{n}^{\circ}$ 6); as mulheres com mais de 30 anos esperam com maior freqüência reduzir peso e melhoria da tonificação geral, com 27,8\% em ambos objetivos.

Na tabela 5, em cada objetivo pode ser observado que o maior percentual de sujeitos é representado por mulheres de 20 a 30 anos querendo reduzir peso $(53,4 \%)$; para aumento de massa muscular, $84,2 \%$ de homens entre 20 e 30 anos; na tonificação geral mulheres de 20 a 30 anos optam em $65,4 \%$ dos casos; no aspecto saúde $33,3 \%$ para cada faixa etária (com exceção de mulheres entre 30 e 40 anos); para o condicionamento físico generalizado as mulheres entre 30 e 40 anos desejam em 35,3\% das avaliações e, para outros objetivos ( $\mathrm{n}^{\circ}$ 6), os homens com menos de 30 anos optam por esta finalidade em $36,4 \%$ dos casos.

$\mathrm{Na}$ mudança de faixa etária a inversão de interesse entre homens e mulheres só não ocorreu na condicionamento físico generalizado e no objetivo 6 (Outros); para o interesse masculino aumento de massa muscular e tonificação geral se reduzem com o passar da idade e aumenta para as mulheres; redução de peso e melhorias na saúde aumentam para os homens e diminuem para as mulheres.

TABELA 4

Distribuição percentual dos praticantes quanto ao objetivo da prática de atividade física em relação a faixa etária e sexo

\begin{tabular}{|c|c|c|c|c|}
\hline \multirow[b]{2}{*}{ Objetivo } & \multicolumn{2}{|c|}{$20-30$} & \multicolumn{2}{|c|}{$30-40$} \\
\hline & $\mathbf{H}$ & $M$ & $\mathbf{H}$ & $\mathbf{M}$ \\
\hline$\downarrow$ Peso & 31,4 & 54,9 & 35,0 & 27,8 \\
\hline$\uparrow$ Massa muscular & 45,7 & 2,8 & 15,0 & 5,6 \\
\hline Tonificação geral & 5,7 & 23,9 & 0,0 & 27,8 \\
\hline Saúde & 1,4 & 1,4 & 5,0 & 0,0 \\
\hline Cond. fís. gen. & 4,3 & 8,5 & 15,0 & 27,8 \\
\hline Outros & 11,4 & 8,5 & 30,0 & 11,1 \\
\hline
\end{tabular}

TABELA 5

Distribuição percentual do sexo e faixa etária em relação a cada objetivo

\begin{tabular}{lrrrrr}
\hline \multirow{2}{*}{ Objetivo } & \multicolumn{2}{c}{ Homens } & & \multicolumn{2}{c}{ Mulheres } \\
\cline { 2 - 3 } & $\mathbf{2 0 - 3 0}$ & $\mathbf{3 0 - 4 0}$ & & $\mathbf{2 0 - 3 0}$ & $\mathbf{3 0 - 4 0}$ \\
$\downarrow$ Peso & 30,1 & 9,6 & & 53,4 & 6,8 \\
$\uparrow$ Massa muscular & 84,2 & 7,9 & & 5,3 & 2,6 \\
Tonificação geral & 15,4 & & & 65,4 & 19,2 \\
Saúde & 33,3 & 33,3 & & 33,3 & \\
Cond. fís. gen. & 17,6 & 17,6 & & 35,3 & 29,4 \\
Outros & 36,4 & 27,3 & & 27,3 & 9,1 \\
\hline
\end{tabular}

\section{DISCUSSÃO}

Quando nas tabelas 1 e 2 são analisadas as médias dos grupos, os indicadores IMC e $\% \mathrm{G}$ podem ser classificados normalmente ${ }^{12-14}$, ou seja, IMC entre 20 e $25 \mathrm{~kg} / \mathrm{m}^{2}$ e $\% \mathrm{G}$ para homens entre 13 e $17 \%$ e para mulheres entre 23 e $27 \%$. Porém, as médias de valores por faixa etária não diferiram de outros estudos ${ }^{6-8,15}$. Na análise por faixa etária e sexo (tabela 3), ficou evidenciado que no aumento de idade estas freqüências não se comportam de maneira igual. Pode ser observado que as mulheres apresentam valores relativos mais próximos dos ideais para os indicadores de saúde, sendo que a maioria delas está na faixa ideal ou abaixo dos valores indicados. Com o passar dos anos os sujeitos aumentam seu IMC e \% G extrapolando com maior frequiência os valores desejados, exceção no $\% \mathrm{G}$ para homens, que se manteve igual, isso pode ser explicado porque homens e mulheres após 35 anos de idade tendem a ganhar mais gordura corporal, até a quinta ou sexta décadas de vida ${ }^{15}$. Assim, o excesso de peso apresentado acima dos 25 anos de idade seria um fator de risco de doenças crônico-degenerativas em ambos os sexos, independentemente do peso inicial ou de outros fatores, conforme sugerem Pollock e Wilmore ${ }^{16}$.

O que de fato chamou a atenção foram os valores da distribuição dos praticantes (tabela 3), que apresentaram decréscimo da condição ideal de saúde - analisada através de indicadores, \%G e IMC - esses valores indicam que as alterações inerentes ao avanço da idade realmente ocorrem e são detectadas por indicadores que utilizam inúmeras variáveis.

Embora maior percentual de mulheres esteja interessado em redução de peso, estas não necessitam tanto quanto os homens; existe um aumento da freqüência de sujeitos que extrapolam as faixas de $\% \mathrm{G}$ e IMC com o avanço da idade. Nota-se ainda que os homens têm maior variação nos indicadores antropométricos e de composição corporal que as mulheres.

Neste estudo aproximadamente 55\% (tabela 4) das mulheres entre 20 e 30 anos procuram o exercício físico como forma de redução do peso corporal, porém, somente 16,9\% delas realmente necessitariam; esta maior procura deveria ocorrer com as mulheres de mais idade, pois $44,4 \%$ deste grupo está com o \% G e IMC acima do indicado. Para Gaes$\operatorname{ser}^{13}, 40 \%$ e $25 \%$, respectivamente de mulheres e homens tentam reduzir seu peso, o que vem de encontro aos valores deste estudo. Um dos fatores que podem vir a explicar este grande interesse pela redução de peso corporal é a mídia, que impõe padrões anoréxicos de estética corporal feminina, sendo as mulheres com menos idade mais sugestionáveis a estes comportamentos. 
A redistribuição dos objetivos (tabela 4) entre as faixas etárias evidencia que a mudança de grupo social influencia os objetivos desses praticantes. Os homens buscam maior condicionamento físico generalizado e a atividade física como forma de lazer, reduzindo desta a preocupação com a estética (representada pelo desejo do aumento de massa muscular) tão realçada entre 20 e 30 anos. No grupo feminino a preocupação com a redução de peso diminui de um grupo etário para outro e novamente cresce a participação na atividade física objetivando condicionamento físico generalizado. Para Dishman ${ }^{17}$, o processo de adoção ao exercício tem relação com as crenças sobre os benefícios da atividade física, mesmo esta estando associada ao bemestar, prazer e sucesso em determinados grupos, entre outras razões.

A redução no nível de participação das pessoas praticantes de atividade física pode ser explicada por inúmeros motivos, sendo a expectativa de benefícios, crédito de profissionais, atitudes e intenções alguns destes ${ }^{18}$; também os componentes dos programas, os segmentos populacionais e a seleção dos exercícios são outros fatores ${ }^{19}$; por fim, o

\section{REFERÊNCIAS}

1. American College of Sports Medicine. Programas adequados e inadequados para redução de peso. (Posicionamento Oficial). Rev Bras Med Esporte 1997;3:125-30.

2. Paffenbarger RS, Hyde MA, Wing AL, Lee I-M, Jung DL, Kampert JB. The association of changes in physical-activity level and other life-style characteristics with mortality among men. N Engl J Med 1993;28:53845.

3. Bronstein MD. Exercício físico e obesidade. Rev Soc Cardiol São Paulo 1996;6:111-6.

4. Nunomura M, Andreotti RA, Teixeira LA, Okuma SS. Nível de adesão ao exercício num programa supervisionado. Rev Bras Ativ Fís e Saúde 1997;2:61-6.

5. Freitas-Júnior, IF. Aptidão física relacionada à saúde em adultos. Rev Bras Ativ Fís e Saúde 1998;1:39-48.

6. Petroski EL, Pires-Neto CS. Validação de equações antropométricas para a estimativa da densidade corporal em mulheres. Rev Bras Ativ Fís e Saúde 1995;1:65-73.

7. Petroski EL, Pires-Neto CS. Validação de equações antropométricas para a estimativa da densidade corporal em homens. Rev Bras Ativ Fís e Saúde 1996;1:5-14.

8. Lopes AS, Nahas MV, Duarte MFS, Pires Neto CS. Distribuição da gordura corpórea subcutânea e índices de adiposidade em indivíduos de 20 a 67 anos de idade. Rev Bras Ativ Fís e Saúde 1995;1:15-6.

9. Wartenweiler J, Hess A, Wuest R. Anthropologic and performance. In: Larson LA. Fitness, health and work capacity. International standards for assessment. New York: Macmilam Publising Co., 1974.

10. Petroski EL. Desenvolvimento e validação de equações generalizadas para a estimativa da densidade corporal em adultos. Tese de Doutorado. Santa Maria, UFSM, 1995. horário e a intensidade das aulas e o tipo de turma (mistas ou não) também influenciam o nível de participação ${ }^{4}$. Ainda, pode-se citar que o maior tempo livre e interesse (nível de prioridade) dos mais jovens fazem com que estes tenham maior frequiência nas academias; e a necessidade de participação no mercado de trabalho, com substancial redução do tempo livre pode levar os sujeitos de mais idade a terem que deixar de participar de programas de atividade física.

As alterações na composição corporal e IMC associadas às mudanças de faixas etárias justificam-se entre si, pois todos esses fatores se inter-relacionam e aumentam os fatores de risco para a saúde ${ }^{20}$. Com o aumento da idade as alterações na composição corporal são associadas a maior incidência na mortalidade ${ }^{21}$.

Dentro das limitações deste estudo, podemos concluir que ocorrem significativos incrementos na gordura corporal em ambos os sexos com o avanço da idade; que a análise dos valores médios de \% e IMC indicam que homens e mulheres têm sua condição de saúde agravada e os objetivos mudam conforme a faixa etária.

11. Siri WE. Body composition from fluids spaces and density: analyses of methods. In: Techniques for measuring body composition, Washington, DC: National Academy of Science and Natural Resource Council, 1961.

12. Petroski EL. (organizador). Antropometria: técnicas e padronizações. Porto Alegre: Palotti, 1999.

13. Gaesser GA. Thinness and weight loss: beneficial or detrimental to longevity? Med Sci Sports Exerc 1999;31:1118-28.

14. Baumgartner TA, Jackson AS. Measurement for evaluation in physical education and exercise science. Dubuque, Iowa: Brown \& Benchmarck, 1995.

15. McArdle WD, Katch FI, Katch VL. Fisiologia do exercício: energia, nutrição e desempenho humano. Rio de Janeiro: Guanabara Koogan, 1998

16. Pollock ML, Wilmore JH. Exercício físico na saúde e na doença. Rio de Janeiro: Medsi, 1993.

17. Dishman, RK. Advances in Exercise Adherence. Champaign, Illinois: Human Kinetics, 1994.

18. Dishman RK. Motivating older adults to exercise. South Med J 1994;5 (Suppl):79-82.

19. Dishman RK, Buckworth J. Increasing physical activity: a quantitative synthesis. Med Sci Sports Exerc 1996;6:706-19.

20. Fédération Internationale de Médecine Sportive. A inatividade física aumenta os fatores de risco para a saúde e a capacidade física. Rev Bras Med Esporte 1998;2:169-70.

21. Roche AF, Heymsfield SB, Lohman TG. Human Body Composition. Champaign, Illinois: Human Kinetics, 1996. 